\title{
IRIS Recognition System using Neural Network and Genetic Algorithm
}

\author{
V. Saishanmuga Raja \\ Research Scholar \\ Department of Computer Science \& Engineering, \\ Dr. MGR Educational \& Research Institute \\ University, Chennai.
}

\author{
S.P. Rajagopalan, PhD. \\ Professor Emeritus \\ Department of Computer Applications, \\ Dr. MGR Educational \& Research Institute \\ University, Chennai.
}

\begin{abstract}
In this paper, the author proposes a method for personal identification based on iris recognition using Genetic algorithm and Neural Network. The process of iris recognition consists of localization of the iris region and generation of data set of iris images followed by iris pattern recognition. A Neural Network is used to reduce the low recognition rate, low accuracy and increased time of recovery. Here the genetic algorithm is used to optimize the Neural Networks parameters. The simulation results show a good identification rate and reduced training time.
\end{abstract}

\section{Keywords}

Genetic algorithm, Neural Network, Iris recognition

\section{INTRODUCTION}

The iris became a much explored field. Human iris contains unique and very important information about persons. Related works have been done in the medicine's domain (determination of some possible health conditions [1]), in biometrics domain (identification and recognition of a person [2]) and others. Biometrics plays an important role in public safety and to accurately identify each individual to distinguish them from each other [3]. The traditional methods normally make use of magnetic cards or cards with some bar codes printed on it. The major problem in these types of methods is that the cards should be carried along where ever a person goes. There are many chances of the cards may be stolen or misplaced. Of late there are many instances of replication of finger prints and magnetic cards and their misuse have come into light. The department of cyber crime has a tough time in cracking the cases and tracking the culprits.

The human iris is unique and cannot be duplicated or imitated, since it is impossible to extract an iris image without the knowledge of a person. Iris region is the part between the pupil and the white sclera. This field is sometimes called iris texture. The iris texture provides many minute characteristics such as freckles, coronas, stripes, furrows, crypts, etc [2]. From the birth of a person until death, the patterns of the iris are relatively constant over a person's lifetime $[1,3]$. Because of this uniqueness and stability, iris recognition is a reliable human identification technique. The process of iris recognition consists of iris image capturing, pre-processing, and recognition of iris region in eye image. The iris image preprocessing includes localization. In practice, the identification process starts when an image of the eye from a person located in front of the digital camera is taken. In the process of image acquisition $T x=f(£ x)$, a digital representation of the biometrics $T x$ is obtained from a real biometric $£ x$.

The next step is segmenting the iris image, which identifies the area of interest: the iris texture. This process may include distinctive features and the encoding phase is a process that allows the biometrics' digital template $T x$ to be obtained. The resulting template is compared to those in a database in mode called 1: $N$ to search for the pattern that best matches the template using a decision threshold $t$ which is chosen based on the assumed security strategy. In the simplest case, a minimum-distance classifier can be used for data-mining in the database $D$, which generates the lowest similarity score $s 1 . . N=s(T x, T 1 . . N)$ according to the criterion

$$
\begin{aligned}
& \mathrm{S} \leq t \Rightarrow \text { match } \\
& \mathrm{S}>t \Rightarrow \text { not match }
\end{aligned}
$$

\section{PREPROCESSING AND SEGMENTATION}

After storing the eye images in the database, the images for generic information from the pupil and iris are observed. To extract the exact image of iris the unwanted components around the iris has to be removed. For doing this the author have the following stages in his experiments.

\subsection{Iris Segmentation}

In preprocessing stage, several methods were implemented for feature extraction and noise removal in order to extract the iris from the captured image. In the process of segmentation, it may be necessary to use certain algorithms whose task is to further prepare the image for processing by the feature extraction algorithms.

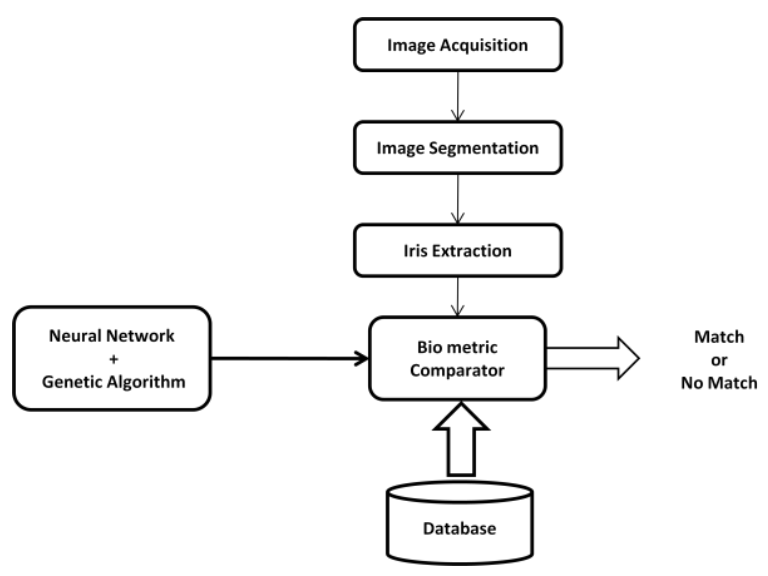

Fig 1: Block diagram of a iris recognition system

The author have modelled the iris boundary as an elliptical surface and used Daughman's Integro-differential Operator (eq.1) [2] to segment the iris portion accordingly.

$$
\left(\mathrm{r}, \mathrm{x}_{\mathrm{c}}, \mathrm{y}_{\mathrm{c}}\right)=\underset{(r, x i, y i)}{\max }\left|\mathrm{G} \sigma(\mathrm{r}) * \frac{\partial}{\partial r} \oint \frac{\mathrm{I}(x, y)}{2 \pi r} \mathrm{ds}\right| \ldots
$$


A coarse search is done to extract the approximate pupil image and a fine search is done to the obtained pupil for an exact iris image. The upper and lower part of the pupil image is discarded to avoid the effects of eye lashes and eye lids.

\subsection{Iris Normalization}

After segmentation certain portions of the circular iris is discarded in order to avoid occlusion [4]. This step is often necessary, since almost in all the images an occluded iris is encountered. After getting the non concentric outer and inner circular shapes of the iris, the circular shape of the iris image can now be converted into a rectangular image by normalizing the iris image. The transformation can be done as shown below

$\mathrm{x}(\mathrm{r}, \theta)=(1-\mathrm{r}) \mathrm{x}_{\mathrm{p}}(\theta)+\mathrm{rx}_{\mathrm{s}}(\theta)$

$\mathrm{y}(\mathrm{r}, \theta)=(1-\mathrm{r}) \mathrm{y}_{\mathrm{p}}(\theta)+\mathrm{ry}_{\mathrm{s}}(\theta)$

Functions $x(r, \theta)$ and $y(r, \theta)$ are defined by a linear combination of coordinates of points on the border of the pupil $(x p(\theta), y p(\theta))$ and coordinates of points on the external border of the iris $(x s(\theta), y s(\theta))$, according to[2].

\subsection{Iris Feature Extraction}

The iris biometric $£$ can be analyzed in various ways to obtain its digital template $T$. In all cases, solutions are based on iris texture filtering, but they can vary significantly. The most recognized approach is the phase-based method proposed by Daugman, which has been successfully used in the majority of available iris recognition systems. The main disadvantage of this approach is that the lighting conditions are not considered while feature extraction. But here it is assumed that the lighting conditions do not have much effect on the feature extraction. Also, this method does not require a complex and expensive vision system. This methodology, which is described in detail in [5], is very reliable and efficient. Therefore, many alternative solutions have been developed for iris feature extraction. The best-known techniques use spatial transformation of the iris texture and correlation of templates, as suggested by Wildes [6]; "zerocrossings wavelet representation," as proposed by Boles and Boashas [7].

\subsection{Biometric Comparator}

To compare the extracted iris image and the image that is stored in the database they use a biometric comparator. The similarity between the two binary images can be calculated in many ways. They use the most recognized method by measuring the Hamming distance[2]. This method works by performing a logical XOR operation on the two binary representations and calculating the number of ones in the vector denoting the non compliance in some positions to the size of $\mathrm{N}$ vectors.

$s=s(T a, T b)=\frac{1}{N} \sum_{j=1}^{N} \mathbf{T a}(\mathbf{j}) \oplus \mathbf{T b}(\mathbf{j})$

This formula allows for very value of $\mathrm{S}$ calculated, which can be further used by processing operations to overcome the problems of unwanted elements such as ambient light reflections, eyelids and eyelashes.

\section{NEURAL NETWORK BASED MODEL}

An Artificial Neural Network (ANN) is an information processing system that is inspired by the way biological nervous system works, such as the brain, process information.
The key element of this paradigm is the structure of the information processing system. It consists of a large number of highly interconnected processing elements (neurons) work to solve specific problems. ANNs, like people, learn by example. An ANN is configured for a specific application, such as pattern recognition or data classification, through a learning process. Learning in biological systems involves adjustments to the synaptic connections that exist between the neurons.

A neural network is a system of parallel processors connected together as a directed graph. Schematically, each processing element (neuron) of the network is represented as a node. These connections provide a hierarchical structure trying to emulate the physiology of the brain for processing new models to solve specific problems in the real world. What is important in developing neural networks is their useful behavior by learning to recognize and apply relationships between objects and patterns of objects specific to the real world. In this respect neural networks are tools that can be used to solve difficult problems [8], [9], [10]. Artificial neural networks are inspired by the architecture of the biological nervous system, which consists of a large number of relatively simple neurons that work in parallel to facilitate rapid decision-making [11].

The ANN is a universal computation algorithm that has the ability to compose complex hypotheses that can explain a high degree of correlation between features without any prior information from the data set (Cartwright, 2008a)

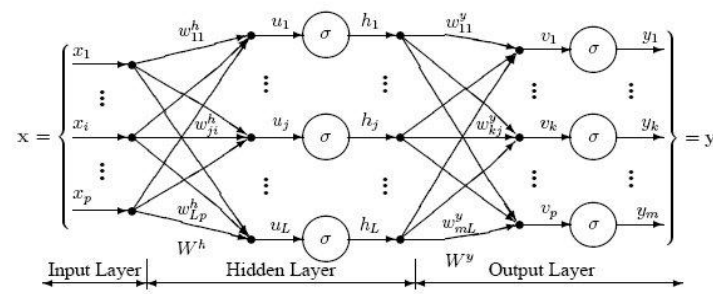

Fig.2 A Neural Network with Hidden layers

A neural network is created and the iris images are given as the inputs. The nodes in the hidden layers of the neural network have to be optimized by the genetic algorithm so that it learns from the given inputs. Here the Artificial Neural Network has to compare the scanned iris image with the stored image and identify the person from the image. For doing this the nodes in the neural network has to learn and store the features of the iris each time an image is being given as input. Aim of this study is to make Neural Network learn faster and accurately identify the person. In some cases if the nodes are forced with a mass data, the network instead of learning may adopt rote learning or memorize. This is just like the children resort to repeat what they read from the book verbatim from their memory instead of acquiring the abstract of the concept and interpret in their own way.

\subsection{Input Layer}

The input layer or the processing stage before the input layer standardizes the input values so that the range of each values is -1 to 1 . The predictor variable values $\left(\mathrm{x}_{1} \ldots \mathrm{x}_{\mathrm{n}}\right)$ is given as input values. Then the values are distributed to each neurons of the hidden layer. In addition to the predictor values, a constant input of 1.0 is presented to each hidden layers which is called as bias. The bias is multiplied by a weight and added to the sum of values going into the neuron. 


\subsection{Hidden Layer}

The value from each input neuron is multiplied by a weight $\left(w_{i j}\right)$, and the resulting weighted values are added together giving a combined value $u_{j}$. Then the combined value $\left(u_{j}\right)$ is put into a transfer function $\sigma$ which gives a output value $h_{j}$. The output from the hidden layer is given to the output layer.

\subsection{Output Layer}

Given a neuron in the output layer, the outputs from each hidden layer neurons are multiplied by a weight $\left(w_{k j}\right)$ and the resulting weighted values are added together producing a combined value $V_{j}$. Then the weighted sum $\left(V_{j}\right)$ is fed into a transfer function, $\sigma$, which gives an output value $y_{k}$. The values are considered as the outputs of the network.

Based upon the concept of regression analysis, when a continuous value is taken as a target variable, then there is a single neuron in the output layer. For categorical target variables in classification problems, there will be $\mathrm{N}$ neurons in the output layer and will produce $\mathrm{N}$ values for each categories of the target variable.

\subsection{Training a Multilayer Neural Network}

The goal of the training process is to find the set of weight values that will cause the output from the neural network to match the actual target values as closely as possible. There are several issues involved in designing and training a multilayer neural network:

- $\quad$ Selecting how many hidden layers to use in the network.

- Deciding how many neurons to use in each hidden layer.

- $\quad$ Finding a globally optimal solution that avoids local minima.

- Converging to an optimal solution in a reasonable period of time.

- Validating the neural network to test for over fitting.

\subsection{Selecting the Number of Hidden Layers}

For most of the problems, one hidden layer is enough. For modeling data with discontinuities such as saw tooth patterns may require two hidden layers. Using two hidden layers may rarely improve the model.

\subsubsection{Deciding how many neurons to use in the hidden layers}

One of the most important characteristics of a neural network is the number of neurons in the hidden layers. If less number of neurons are used, the network may not be able to model complex data and the result will be very poor. If too many neurons are used, then the time taken to train the network may become long and worse. Over fitting may also occur due to excessive neurons and the network may begin to model random noise in the data. As a result the model may fit training data well but may behave poorly to the unseen data.

\section{GENETIC OPTIMIZATION}

As a preparation to start the optimization process, a Genetic Algorithm, requires a group of initial solutions as the first generation. The first generation is usually a group of randomly produced solutions created by a random number generator. The population, which is the number of individuals in a generation, should be big enough so that there could be a reasonable amount of genetic diversity in the population. Also, it should be small enough for each generation to be computed in a reasonable period of time using the computer resources available. Typically, a population includes individuals between 20 and 100 .

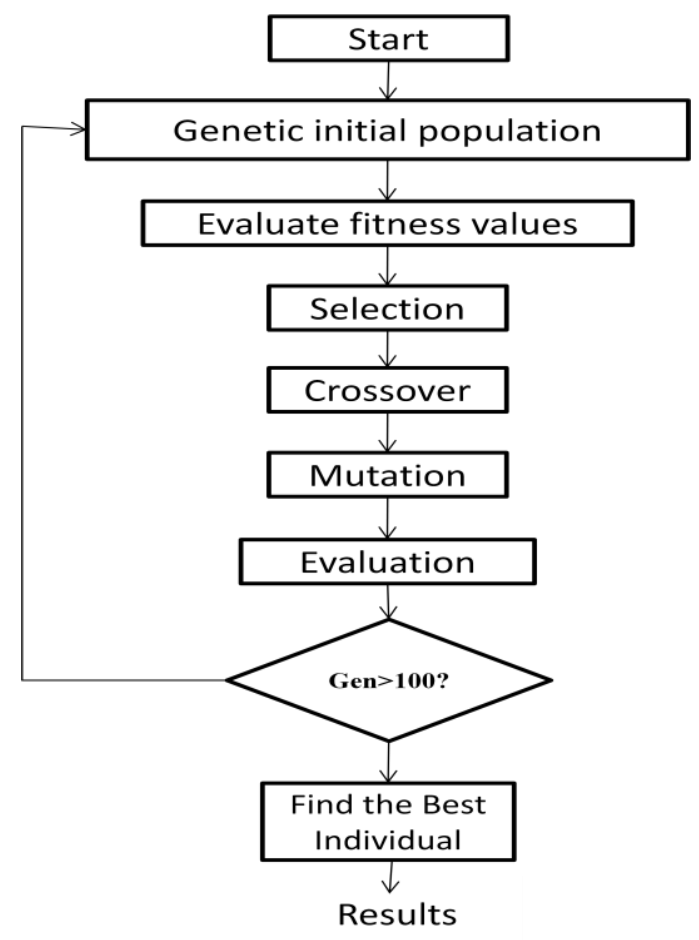

Fig.3. Flowchart of a simple Genetic Algorithm

The fitness function is evaluated to measure how close that the individuals fit the desired result. A fitness function could be either complex or simple depending on the optimization problem addressed. In a case of minimization problem, the most fitted individuals will have the lowest numerical value of the associated fitness function.

Individuals are selected according to a fitness-based process. The operator of selection is made up of ranking and selection progress, by which more copies of the individuals that fit the optimization problem better will be produced in the next generation. In GAs, there are mainly two ways to select a new population: Roulette Wheel Selection (RWS) and Stochastic Universal Sampling (SUS). The individuals will be recombined (crossover) after the selection. This operation is to produce two new individuals from two existing individuals selected by the operator of selection by cutting them at one or more position and exchanging the parts following the cut. The new individuals therefore can inherit some parts of both parents' genetic material. There are usually four ways of doing this: one point crossover, two-point crossover, cycle crossover and uniform crossover (Pham and Karaboga 1998). Figure 4 (a) shows an example of the two-point crossover progress. Mutation is another operator to produce new individuals. 
The difference is that the new individual is produced from a single old one. In this operation, the bit values of each individual are randomly reversed according to a specified property. A mutation can also helps the GA to avoid local optimums and find the global best solution. Figure 4 (b) represents how the mutation operator works.

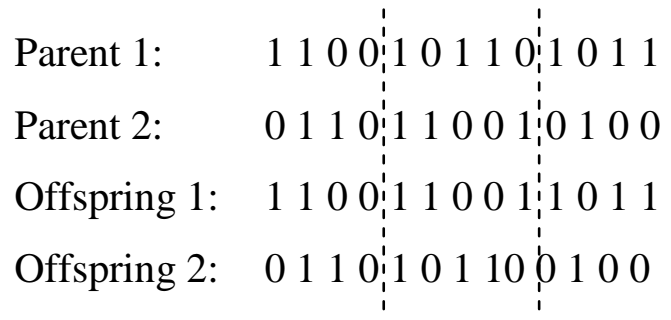

(a)

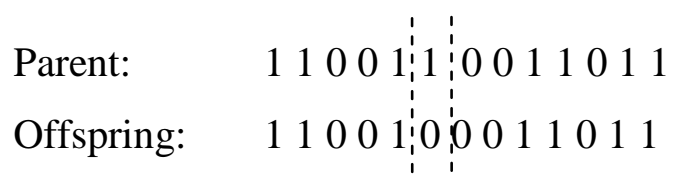

(b)

Figure 4 (a) Crossover operation; (b) Mutation operation

Then the fitness value of each individual in the second generation is computed again. This cycle will not stop until the result is close enough or after a certain generation. GA was run 10 times to find the standard deviation and average of the results of the neurons, methods and number of layers. The report of the training behavior of the GA is obtained with the results of the 10 experiments and it is used to compare the results of the other approaches. The parameter of the chromosomes that were used in the GA are shown in the table 1. The real chromosome is composed of 3 layers and each layer is composed of 300 neurons, which varies in the range of 0 to 300 values and 4 training models. The above said experiment was done in MATLAB version 7.5.The training methods are shown below.

Table 1. Parameters of the chromosome for the GA

\begin{tabular}{|c|l|l|l|c|}
\hline Module & $\begin{array}{l}\text { Layer1 } \\
\text { (neurons) }\end{array}$ & $\begin{array}{l}\text { Layer2 } \\
\text { (neurons) }\end{array}$ & $\begin{array}{l}\text { Layer3 } \\
\text { (neurons) }\end{array}$ & Method \\
\hline M1 & $0 \ldots \ldots 300$ & $0 \ldots . .300$ & $0 \ldots . .300$ & $1: 4$ \\
\hline M2 & $0 \ldots . .300$ & $0 \ldots . .300$ & $0 \ldots . .300$ & $1: 4$ \\
\hline M3 & $0 \ldots \ldots 300$ & $0 \ldots . .300$ & $0 \ldots . .300$ & $1: 4$ \\
\hline
\end{tabular}

Table 2. Training methods for the GA

\begin{tabular}{|l|l|}
\hline \multicolumn{2}{|c|}{ Selected Methods } \\
\hline Trainscg & Scaled conjugate \\
\hline Traingdm & Gradient descent with momentum \\
\hline Traingdx & $\begin{array}{l}\text { Gradient descent with momentum and adaptive } \\
\text { learning factor }\end{array}$ \\
\hline Traingda & Gradient descent with adaptive learning factor \\
\hline
\end{tabular}

The results of the GA after 10 runs for the 3 modules are shown in the table 3 , table 4 , and table 5 .
Table 3. Results of GA for module 1

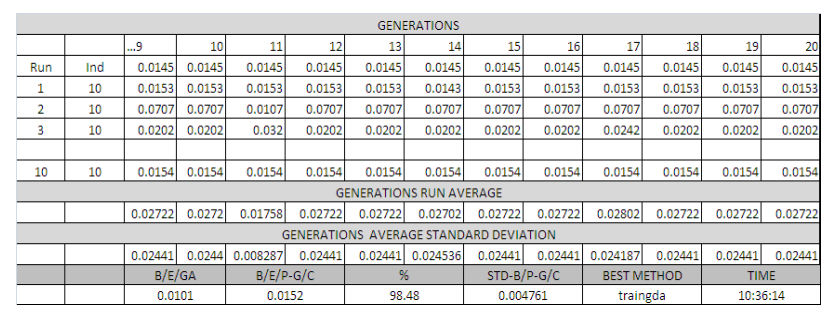

Table 4. Results of GA for module 2

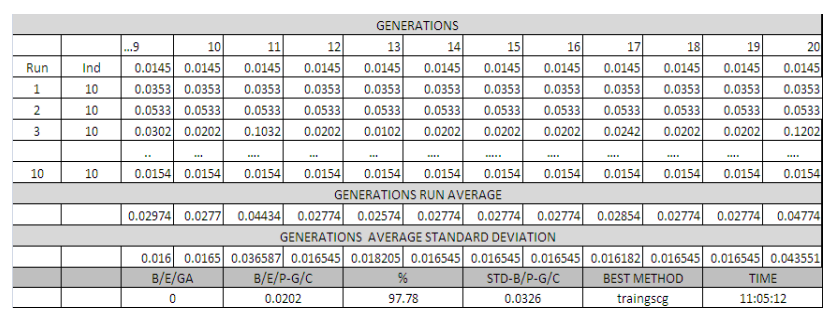

Table 5. Results of GA for module 3

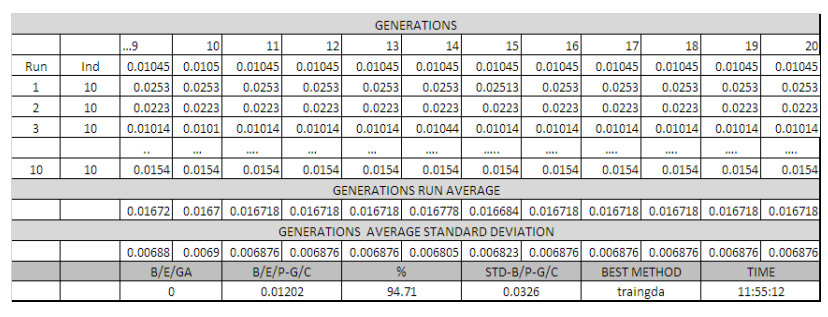

The above results show the training performed with Neural network optimized by Genetic algorithm with 20 generations, 10 runs, and 10 individuals. Showing the average in each generation and standard deviation for each generation run, better error found by genetic algorithm, best training method and execution time.

Table 6. Recognition performance comparison with the existing methods

\begin{tabular}{|l|c|c|}
\hline Methodology & Accuracy Rate & Average time \\
\hline Daugman [5] & $100 \%$ & $90 \mathrm{~s}$ \\
\hline Boles [12] & $92.64 \%$ & $110 \mathrm{~s}$ \\
\hline $\begin{array}{l}\text { Neural network } \\
\text { without GA }\end{array}$ & $93.3 \%$ & $20 \mathrm{~s}$ \\
\hline $\begin{array}{l}\text { Neural network } \\
\text { with GA }\end{array}$ & $98.48 \%$ & $10.8 \mathrm{~s}$ \\
\hline
\end{tabular}

The results show that the genetic optimized neural network outperforms the other approaches like BP in the means of learning time, and high accuracy in identifying the iris images with the persons.

\section{CONCLUSION}

A Genetic algorithm optimization of neural network for human iris recognition system has been proposed in this paper. The proposed method uses genetic algorithm for optimization of the existing neural network method to achieve high accuracy and reduce learning time. From the result it is evident that the proposed method is much better in terms of accuracy in iris recognition and less error rate. Compared with 
the traditional neural network, the proposed method achieved better accuracy rate when it is optimized by the genetic algorithm.

Although the results are encouraging, the network in the initial stages started mugging up the results instead of learning and predicting. This issue is to be dealt as a future work.

\section{REFERENCES}

[1] Demea S., "Corelatii intre aspectul irisului si patologia generala" (Correlations between iris aspects and endocrine pathology), PhD Thesis, manuscript sent for publications in Sibiu Medical 2005.

[2] John Daugman, "How Iris Recognition Works", IEEE Transactions on Circuits And Systems For Video Technology, Vol. 14, No. 1, January 2004

[3] Melin P, Castillo O. Hybrid Intelligent Systems for Pattern Recognition. Heidelberg: Springer-Verlag. 2005.

[4] S. Mukerjee, s. Chandha, "A Robust Human Iris Verification Using a Novel Combination of Features" IEEE explore.

[5] J. Daugman, "High confidence visual recognition of persons by a test of statistical independence," IEEE Trans. Pattern Anal. Mach. Intell., vol. 15, no. 11, pp. 1148-1161, Nov. 1993.

[6] R. Wildes, "Iris recognition: An emerging biometric technology," Proc.IEEE, vol. 85, no. 9, pp. 1348-1363, Sep. 1997.

[7] W. Boles and B. Boashash, "A human identification technique using images of the iris and wavelet transform," IEEE Trans. Signal Process., vol. 46, no. 4, pp. 1185-1188, Apr. 1998
[8] Fallahnezhad M, Moradi MH, Zaferanlouei S. A Hybrid Higher Order Neural Classifier for handling classification problems, Expert Systems with Applications. 2011; 38(1): 386-393.

[9] Sibai FN, Hosani HI, Naqbi RM, Dhanhani S, Shehhi S. Iris recognition using artificial neural networks, Expert Systems with Applications. 2011; 38(5): 59405946.

[10] Wade JJ, McDaid LJ, Santos JA, Sayers HM. SWAT: A spiking neural network training algorithm for classification problems, IEEE Transactions on Neural Networks. 2010; 21(11): 1817-1830.

[11] Zeng J, Liu ZQ. Type-2 fuzzy hidden Markov models to phoneme recognition, Proceedings of the International Conference on Pattern Recognition (ICRP), Cambridge 2004: 192-195.

[12] W. Boles and B. Boashash, "A Human Identification Technique Using Images of the Iris and Wavelet Transform," IEEE Trans. Signal Processing, vol. 46, no. 4, pp.1185-1188, 1998

[13] P. Kronfeld, "Gross anatomy and embryology of the eye," in The Eye, H. Davson, Ed. London, U.K.: Academic, 1962.

[14] Rahib H.Abiyev, Koray Altunkaya, "Personal Iris Recognition Using Neural Network", International Journal of Security and its Applications Vol. 2, No. 2, April, 2008.

[15] Patricia Melin*, Victor Herrera, Danniela Romero, Fevrier Valdez, Oscar Castillo, "Genetic Optimization of Neural Networks for Person Recognition Based on the Iris", TELKOMNIKA, Vol.10, No.2, June 2012, pp. 309 320. 